\title{
Quality of Life and Health Status among Prostate Cancer Survivors and Non-cancer Population Controls
}

\author{
Lixin Song, $\mathrm{PhD}^{1,2}$, Yingchun Ji, $\mathrm{PhD}^{3}$, and Mathew E. Nielsen, MD, MS ${ }^{1,4}$ \\ [1] School of Nursing, University of North Carolina (UNC), Chapel Hill, NC \\ [2] Lineberger Comprehensive Cancer Center, UNC, Chapel Hill, NC \\ [3] Asia Research Institute, National University of Singapore, Singapore \\ $\left[{ }^{4}\right]$ Division of Urologic Surgery, School of Medicine, UNC, Chapel Hill, NC
}

\begin{abstract}
Objective: To examine whether quality of life (QOL), health status, and the relationships between them varied by having a prostate cancer history. This study helps to inform the interface between aging-related health decline and the survival state among older men with prostate cancer, which is an important yet understudied public health issue.
\end{abstract}

Methods: Hierarchical linear models were used to analyze the cross-sectional data from the nationally representative population-based Medical Expenditure Panel Survey. Using propensity score matching, survivors (respondents with prostate cancer history) and controls (respondents without a history of any cancer) ( $\mathrm{N}=193$ pairs) were matched based on seven socio-demographic and health-related factors. QOL was measured using the mental and physical component scores of the SF12. Health status included comorbidities, activities of daily living (ADL), instrumental activities of daily living (IADL), and depressed mood.

Results: In bivariate analyses, survivors reported worse physical (42,72 vs 45.45 respectively, $\mathrm{p}=.0040$ ) and mental QOL (51.59 vs 53.73 respectively, $\mathrm{p}=0.0295)$ and more comorbidities (3.25 vs 2.78 respectively, $\mathrm{p}=0.0139$ ) than controls. In multivariate analyses, for both survivors and controls, better physical QOL was associated with fewer comorbidities ( $<<0.0001)$, no need help with ADL ( $\mathrm{p}=0.0011)$ and IADL ( $\mathrm{p}=0.0162)$, and less depressed mood ( $\mathrm{p}<0.0001)$; better mental QOL was associated with no need help with IADL ( $\mathrm{p}=.0005)$ and less depressed mood $(\mathrm{p}<0.0001)$.

Conclusions: QOL of older men is affected by physical, functional, and psychological factors rather than by prostate cancer history. Clinicians need to attend to aging-related health issues when providing care for prostate cancer survivors to improve QOL.

\section{Keywords}

quality of life; comorbidity; activity of daily living (ADL); depressed mood; propensity score; prostate cancer

(C) 2013 Elsevier Inc. All rights reserved

Correspondence author: Lixin Song, University of North Carolina-Chapel Hill, Carrington Hall, Chapel Hill, NC 27599-7460. Tel: 919-966-3612. Fax: 919-843-9900. 1song@unc.edu.

Publisher's Disclaimer: This is a PDF file of an unedited manuscript that has been accepted for publication. As a service to our customers we are providing this early version of the manuscript. The manuscript will undergo copyediting, typesetting, and review of the resulting proof before it is published in its final citable form. Please note that during the production process errors may be discovered which could affect the content, and all legal disclaimers that apply to the journal pertain.

Support/Financial Disclosures: None. 


\section{INSTRODUCTION}

Cancer survivors are at an increased risk for age-related declines in health status physically, psychologically, and functionally. ${ }^{1}$ Better physical and mental QOL has been associated with less comorbidity among cancer survivors, especially those who survive five years and longer. ${ }^{2}$ Some population-based studies have revealed that, compared to individuals without cancer, cancer survivors have more decrements in self-rated health, ${ }^{3-5}$ psychological wellbeing, and activities of daily living (ADL) $;{ }^{4,6}$ more comorbidities, ${ }^{2}$ higher levels of lost productivity; ${ }^{3-5}$ and similar quality of life (QOL). ${ }^{6}$

Although these population-based studies provided insights regarding physical, functional, and psychological status and QOL during cancer survivorship, further examination is warranted because of several methodological limitations. First, these studies included a collection of survivors of multiple cancer sites, including both genders and with a wide age range (from 18 to $65+$ years of age) ${ }^{2-6}$ Members of such a heterogeneous population differ significantly in their physical, emotional, social, and functional issues and QOL challenges, which make it difficult to draw definitive conclusions regarding health status, QOL, and the association between them. Second, these studies examined the differences in health status between cancer survivors and non-cancer populations ${ }^{4-6}$ or how QOL was affected by one aspect of their health status (e.g., comorbidities). ${ }^{2}$ Little research has examined how QOL is cumulatively affected by the overall health status of cancer survivors, especially aging survivors who are challenged by functional impairment, various comorbidities, and psychological problems. ${ }^{7,8}$ Third, these studies either did not examine QOL, ${ }^{3,4}$ one of the most important patient-reported outcomes, or used a single-item measurement ${ }^{6}$ or a set of proxy measures ${ }^{5}$ instead of a comprehensive assessment of QOL. Finally, although some studies used non-cancer controls, ${ }^{2-4}$ they used one or two matching variables (e.g., age), and thus, the non-cancer population significantly differed from the cancer population in the composition of age groups, marital status, education levels, ${ }^{2,4}$ health insurance coverage ${ }^{4}$, and racial/ethnic background. ${ }^{2-4}$ It is unclear whether variations in study participants' sociodemographic status have contributed to the observed differences in the health outcomes between survivors and non-cancer controls.

To address the aforementioned limitations in population-based research, we investigated the overall health status (i.e., including functional impairment, comorbidities, and psychological problems), QOL, and the association between health status and QOL among prostate cancer survivors (survivors) and matched controls without cancer (non-cancer controls).

Improvements in early detection and treatment have reduced prostate cancer-specific mortality and increased the number of patients who survive for an extended period of time. Yet men with prostate cancer often face physical and psychological morbidities while managing the effects of prostate cancer and its treatment, ${ }^{9}$ which negatively affect the multidimensional aspects of QOL including physical and mental QOL. ${ }^{10,11}$

Furthermore, prostate cancer is most prevalent among older men; the number of prostate cancer survivors will continue to rise as the population ages. ${ }^{12}$ The interface between prostate cancer survivorship and the consequences of aging, including general health decline, is becoming an increasingly important public health issue. Yet there is limited evidence about whether prostate cancer survivors differ from the non-cancer general population in health status, QOL, and the relationships between QOL and health status. Using population-based national survey data and rigorous propensity score matching, we examined (1) whether prostate cancer survivors who are 50 years and older had significantly worse QOL and health status (functional status, comorbidities, and depressed mood) than non-cancer matched controls; and (2) whether QOL was significantly related to health status 
variables in survivors and non-cancer controls. The results from this study provide empirical evidence for comprehensive clinical care for prostate cancer survivors.

\section{MATERIAL AND METHODS}

\section{Sample and propensity matching}

The study sample, drawn from the 2009 Medical Expenditure Panel Survey (MEPS), ${ }^{13}$ included prostate cancer survivors and their matched controls without any cancer history. MEPS is a nationally representative survey of health care utilization and expenditures in the U.S. ambulatory population. ${ }^{13}$ This study focused on survivors who had a prostate cancer diagnosis and who were 50 years of age or older at the time of the 2009 MEPS survey.

To reduce bias and increase estimation precision in this observational study,, ENREF $19^{14}$ we used rigorous propensity score matching to balance the large heterogeneities between the survivor and control groups. A propensity score is a conditional probability that an individual is in one condition rather than in another, ${ }^{14}$ e.g., having a history of prostate cancer. In this study, we first identified, among the 36,855 MEPS respondents, 3,093 male respondents who either had a history of prostate cancer $(\mathrm{N}=193)$ or no cancer at all; were 50 years or older; were of any of the following racial groups: non-Hispanic Whites, Hispanics, or African/Black Americans; and had no missing values in the matching covariates and variables of interest. We then conducted a Stata PSMATCH2; the full Mahalanobis matching and the nearest neighbor matching strategies ${ }^{14}$ were employed to match each of the survivors with one male respondent without a history of any type of cancer based on seven characteristic factors (see below). Finally, we obtained 193 matched pairs of survivors and controls who had closest propensity scores, and thus, attained a random sample of the non-cancer control candidates to match each prostate cancer survivor.

Matching covariates included seven factors that have been linked to QOL and health status among prostate cancer survivors: ${ }^{15-17}$ race (i.e., non-Hispanic Whites, Hispanics, and African Americans); age; education; marital status (married vs. not married); health insurance (having any private, public only, or no insurance); family income; and Body Mass Index (BMI). ${ }^{15-17}$ Regarding age, five-year intervals (50-54, 55-59, 60-64, 65-69, 70-74, or $\geq 75$ years) were used. Educational attainment was categorized into two groups, i.e. shigh school or $\geq$ some college. Family income was measured according to its relationship to the poverty line as reported by U.S. Census Bureau in $2009^{18}$ (adjusted by family size and composition): low ( $<200 \%$ of the poverty line), middle (200\% 400\%), and high income $(>400 \%)$. Lifestyle is another factor that contributes to prostate cancer and other health problems. ${ }^{15} \mathrm{BMI}$, used as a proxy of lifestyle and one of the matching factors, was collapsed into 3 groups $(<25,25 \sim 30$ and $\geq 30)$ because less than $1 \%$ of participants reported a BMI $\leq 8.5$.

\section{Measures}

The outcome variable, QOL, was measured using the SF- $12{ }^{\circledR}$ Health Survey (version 2.0), a validated shortened version of SF-36. ${ }^{19}$ MEPS provides the physical and mental component scores which range from 0 to 100 . With the population means at 50, higher scores indicate better physical and mental QOL, respectively. ${ }^{19}$

The health status variables included functional impairments, the number of comorbid conditions, and depressed mood. Higher values of these measures indicated having functional impairment, more comorbidities, and worse depressed mood. Functional impairments were measured using activities of daily living (ADL) and instrumental activities of daily living (IADL). The ADL referred to whether the respondent needed help or supervision with daily activities such as eating, bathing, and dressing. The IADL 
indicated the respondent's needs for assistance with activities such as taking medications, doing laundry, and going shopping. The need for help and supervision for ADL and IADL was coded as 1 and no need for help as 0 .

Comorbidities in MEPS were measured using a list of conditions that have relatively high prevalence and for which generally accepted standards for appropriate clinical care have been developed. ${ }^{13}$ The respondents were asked whether they had been diagnosed with major diseases since age 18 such as diabetes, hypertension. The respondent's having or not having a diagnosis was coded as 1 or 0 , respectively. The comorbidity index was the sum of values of all comorbid conditions. Depressed mood was measured by Patient Health Questionnaire (PHQ-2) ${ }^{20}$ (having little interest or pleasure in doing things; feeling down, depressed, or hopeless during the past two weeks).

\section{Data Analysis}

The matching covariates, QOL, and health status variables between survivors and noncancer controls were first compared to examine whether there was significant post-matching difference between the two groups. SAS PROC GENMOD was used to account for clustering effects due to pairing survivors and non-cancer controls. To achieve research aims while accounting for the clustering effects, a series of hierarchical linear models were specified using PROC MIXED (SAS 9.3, Cary, NC) ${ }^{21}$ Specifically, two crude models were first constructed by separately regressing physical and mental QOL with respondents' cancer status (survivor $=1$ vs. control $=0$ ). A series of adjusted models were then specified by adding to the crude models (1) the health status variables; (2) each interaction term between cancer status and health status variables; and (3) all of the interaction terms simultaneously. Any imbalanced covariates between survivor and control were included in model specification.

\section{RESULTS}

\section{Descriptive analysis}

Approximately 50\% of the respondents were 65 years of age or older, non-Hispanic white, married, insured with some types of private insurance, with high school or lower education, with middle to high family income, and with a BMI at 25 or greater. After propensity score matching, the survivors and non-cancer controls did not differ significantly in their race, age, education, marital status, medical insurance status, and BMI (Table 1). Regarding family income, there was no significant difference in the percentages of respondents in the low income category between the two groups. Compared to non-cancer controls, however, a higher percentage of survivors reported having middle incomes [59/193 (31\%) vs. 50/193 $(26 \%) ; p=.0478]$ and a lower percentage of survivors had high income [84/193 (43\%) vs. 96/193 (50\%); $p=.0094]$. Family income, thus, was included in model specification to account for the between group imbalance.

Regarding QOL and health status variables (Table 2), the survivors had lower mean scores of physical QOL ( 42.72 vs. $45.45 ; \mathrm{p}=.0040$ ) and mental QOL (51.59 vs. $53.73 ; \mathrm{p}=.0295$ ) than the controls. This suggests that after balancing the matching covariates, the survivors fared worse in physical and mental QOL than their non-cancer counterparts. The mean number of comorbidities that the survivors reported was significantly higher than that reported by the controls ( 3.25 vs. $2.78 ; \mathrm{p}=.0139$ ). No differences were seen in ADL, IADL and depressed mood.

\section{Model Specification}

Physical QOL and Health Status-The PCS1 model in Table 3 shows that survivors had lower scores in physical QOL than controls. The adjusted model PCS2 that included all 
health status variables indicates that poorer physical QOL was associated with needing help with ADL ( $\mathrm{p}=.0011)$ and IADL ( $\mathrm{p}=.0162)$, and having more comorbidities and worse depressed mood (both ps<.0001) in both groups. The PCS3-6 models separately examined each of the interactions between cancer status and health status variables. All of the health status variables remained significant (data not shown); neither cancer status nor the interaction terms were significant. Thus, interaction terms were dropped off the model specification; only the main effects of the cancer status, ADL, IADL, comorbidities, and depressed mood remained in the final model (PCS2). Family income, controlled as a covariate, was significant in all models (all ps $<.05$ ), suggesting that, compared to those who reported low income, respondents in high income group had better physical QOL.

Mental QOL and Health Status-A similar model specification process was repeated with the dependent variables being replaced by mental QOL (Table 3). In MCS1 model, cancer status had a significant negative effect on mental QOL ( $\mathrm{p}=.0349)$, suggesting that the survivors had poorer mental QOL than controls without accounting for the effects of health status. The MCS2 model examined the main effects of health status variables; better mental QOL was associated with no need for help with IADL ( $\mathrm{p}=.0005)$ and less depressed mood ( $\mathrm{p}<.0001)$ in both groups. The adjusted models MCS3-6 separately examined the effects of the health status variables and the interaction terms between cancer status and each health status variable. The main effects of IADL and depressed mood remained significant (both $\mathrm{p}$ values<.001) (data not shown); the effects of cancer status and each of the interaction terms were not significant. Thus, the interaction terms were dropped off and only the main effects of health status variables remained in the final model (MCS2) in which IADL ( $\mathrm{p}=.0005)$ and depressed mood were significant $(\mathrm{p}<.0001)$. Family income was controlled for its imbalance between groups; the effect of medium income was significant $(\mathrm{p}=.0012)$, suggesting that participants who reported medium income had better mental QOL than those in low and high income groups.

\section{DISCUSSION}

This population-based study examined the physical, psychological, and functional impacts of the interplay of aging and prostate cancer and investigated the collective effects of a set of health status variables on the mental and physical QOL among men 50 years of age and older. We found survivors had lower physical and mental QOL and more comorbidities than non-cancer controls but similar depressed mood. The percentage of individuals needing help with ADL and IADL were similar between the two groups. These results corroborate the evidence that older adult survivors are vulnerable to both cancer-related symptoms and aging-related deterioration in health conditions. ${ }^{22}$

In addition, regardless of respondents' prostate cancer history, better physical QOL was associated with need no help with ADL and IADL, fewer comorbidities, and less depressed mood; better mental QOL was associated with need no help with IADL function and less depressed mood. Previous research has reported that prostate cancer-related physiological and psychological symptoms significantly predicted QOL of men with localized prostate cancer within the first 12 months of diagnosis. ${ }^{23}$ Given that $89 \%$ of the survivors in this study were at least one year out since their cancer diagnosis our findings add to the literature on the integral roles that aging-related physical, psychological, and functional factors play in the QOL in intermediate- and long-term prostate cancer survivors.

The results of this study also suggested the importance of including multiple health status variables in evaluating QOL of prostate cancer survivors. Findings across studies have been inconsistent concerning the differences in mental and physical QOL between cancer survivors and non-cancer populations. ${ }^{24,25}$ Focusing on how cancer status or type affected 
QOL, these studies have paid less attention to how QOL is affected by survivors' health status. In this study, when only respondents' cancer status (i.e., having a history of prostate cancer vs. no cancer) was taken into account, the results showed that survivors fared worse in physical and mental QOL than non-cancer controls who had similar sociodemographic background. However, the effect of cancer status on physical and mental QOL disappeared when multiple health status variables were included in multivariate analyses. Similarly, the relationships between QOL and health status did not vary by respondents' cancer status. These results suggest that, for older survivors, cancer occurs against a background of functional declines and other chronic health conditions. Compared to the history of a prostate cancer diagnosis, respondents' health status variables were more important factors when evaluating QOL for men 50 years and older.

Depressed mood was the only health status variable that negatively affected both physical and mental QOL among these men. Consistent with previous findings, ${ }^{26}$ these results stress the importance of evaluating depressive sequelae in cancer survivorship, because such mental distress is likely treatable; early detection and intervention can improve depressed mood, ${ }^{27}$ and ultimately, improve QOL for prostate cancer survivors. These findings highlight the need to address psychological functioning as an integral aspect of cancer survivorship care.

The number of comorbidities was negatively associated with physical QOL. Consistent with the findings from earlier national surveys of elderly cancer patients, ${ }^{2,4}$ prostate cancer survivors in MEPS reported significantly greater numbers of comorbidities than the noncancer controls. Fewer comorbid conditions have been associated with better physical and mental QOL $^{2}$ and improved estimated life expectancy and quality-adjusted life expectancy in prostate cancer survivors. ${ }^{28}$ Thus, similar to caring for non-cancer populations, attention to the management of comorbid conditions should be an integral element of survivorship care to improve health outcomes for prostate cancer survivors.

Functional status (i.e., ADL and IADL) was significantly associated with different aspects of men's QOL. Consistent with the results of previous population-based studies of communitydwelling people of 50 years and older, ${ }^{29}$ the results of this study indicated that needing no help with ADL and IADL was associated with better physical QOL, and needing no help with IADL was associated with better mental QOL. It is possible that men's needing help with ADL and IADL made their dependence on others more perceivable, which in turn affected their sense of control and self-esteem, resulting in poorer physical and mental QOL. Interestingly, similar percentages of survivors and controls reported needing assistance with ADL and IADL in this study. In contrast, the results of an earlier study ${ }^{4}$ using National Health Interview Survey data reported significantly greater limitations in ADL or IADL in cancer survivors than in respondents without cancer or without other illness. Further research is needed to examine whether the differences in research methods between these studies (i.e., use of rigorously matched non-cancer population and heterogonous survivors of different age and gender and with different types of cancer) have contributed to the differences in the findings.

The following limitations in this secondary analysis warrant acknowledgement. First, the MEPS dataset started to have some cancer-related information in 2009. Yet important variables such as cancer stage and treatment type were not available. Second, due to a lack of cancer-related longitudinal data in MEPS, we used a cross-sectional design which has limited causal conclusions of the relationships between QOL and health status. Finally, depressed mood was measured using the two screening questions of the PHQ-2, which may omit some of the symptomatology required for a clinical diagnosis via the Diagnostic and 
Statistical Manual of Mental Disorders system, ${ }^{30}$ and thus, may have limited sensitivity for clinically significant cases of depression.

Nonetheless, this study of nationally representative population-based survey has used propensity score matching to rigorously control for a series of potential confounders, thus reducing biases and increasing the estimation precision. The results suggest that physical and mental QOL of older men is affected by functional, physical, and psychological health status but is not dominated by a history of prostate cancer. In order to improve QOL for prostate cancer survivors, clinicians must be attentive not only to treating the cancer, but also to addressing patients' ADL and IADL needs, comorbidities, and depressed mood.

\section{Acknowledgments}

The authors gratefully acknowledge the statistical support of Drs. Chris Wiesen at the Odum Research Institute, George Knafl at the School of Nursing, and Mark Weaver at the Department of Statistics. We are also grateful for the editorial support of Drs. Marilyn Oermann at the School of Nursing, Morris Weinberger and Laura Ross Loehr at the School of Public Health, and Ms. Susan Pusek at the North Carolina Translational and Clinical Science Institute, University of North Carolina-Chapel Hill. Dr. Song's work is sponsored by the Clinical and Translational Sciences Award (CTSA), University of North Carolina-Chapel Hill (KL2TR001109 and UL1TR001111 (Runge).

Source of Funding: This project is supported by the University Cancer Research Fund from The University of North Carolina Lineberger Comprehensive Cancer Center.

\section{REFERENCE}

1. Bellizzi K, Mustian K, Palesh O, Diefenbach M. Cancer survivorship and aging : moving the science forward. CA: a Cancer Journal for Clinicians. 2008; 113:3530-9.

2. Smith AW, Reeve BB, Bellizzi KM, Harlan LC, Klabunde CN, Amsellem M, Bierman AS, Hays RD. Cancer, comorbidities, and health-related quality of life of older adults. Health Care Financ Rev. 2008; 29:41-56. [PubMed: 18773613]

3. Yabroff KR, Lawrence WF, Clauser S, Davis WW, Brown ML. Burden of Illness in Cancer Survivors: Findings From a Population-Based National Sample. J. Natl. Cancer Inst. 2004; 96:1322-1330. [PubMed: 15339970]

4. Hewitt M, Rowland JH, Yancik R. Cancer survivors in the United States: age, health, and disability. J Gerontol A Biol Sci Med Sci. 2003; 58:82-91. [PubMed: 12560417]

5. Richardson LC, Wingo PA, Zack MM, Zahran HS, King JB. Health-related quality of life in cancer survivors between ages 20 and 64 years: population-based estimates from the Behavioral Risk Factor Surveillance System. Cancer. 2008; 112:1380-9. [PubMed: 18219664]

6. Eakin EG, Youlden DR, Baade PD, Lawler SP, Reeves MM, Heyworth JS, Fritschi L. Health status of long-term cancer survivors: results from an Australian population-based sample. Cancer Epidemiol Biomarkers Prev. 2006; 15:1969-76. [PubMed: 17035407]

7. Liao Y, McGee DL, Cao G, Cooper RS. Recent changes in the health status of the older U.S. population: findings from the 1984 and 1994 supplement on aging. J Am Geriatr Soc. 2001; 49:443-9. [PubMed: 11347789]

8. Freedman VA, Martin LG, Schoeni RF. Recent trends in disability and functioning among older adults in the United States: a systematic review. JAMA. 2002; 288:3137-46. [PubMed: 12495394]

9. Snyder CF, Frick KD, Blackford AL, Herbert RJ, Neville BA. Carducci MA and Earle CC: How does initial treatment choice affect short-term and long-term costs for clinically localized prostate cancer? Cancer. 2010; 116:5391-9. [PubMed: 20734396]

10. Talcott JA. Prostate cancer quality of life: beyond initial treatment and the patient.[comment]. Journal of Clinical Oncology. 2007; 25:4155-6. [PubMed: 17635949]

11. Litwin MS, Gore JL, Kwan L, Brandeis JM, Lee SP, Withers HR, Reiter RE. Quality of life after surgery, external beam irradiation, or brachytherapy for early-stage prostate cancer. Cancer. 2007; 109:2239-47. [PubMed: 17455209]

Urology. Author manuscript; available in PMC 2015 March 01. 
12. Howlader, N.; Noone, AM.; Krapcho, M.; Neyman, N.; Aminou, R.; Waldron, W.; Altekruse, SF.; Kosary, CL.; Ruhl, J.; Tatalovich, Z. SEER Cancer Statistics Review, 1975-2008. Institute. NC; Bethesda, MD: 2011.

13. Center for Financing A. Cost Trends. MEPS HC-129 2009 Full Year Consolidated Data File, in Agency for Healthcare Research and Quality. Rockville, MD: 2009.

14. Guo, SY.; Fraser, MW. Propensity Score Analysis: Statistical Methods And Applications. CA, Sage Publications; California: 2009.

15. American Cancer Society. Cancer Facts \& Figures 2011. 2011

16. Song L, Northouse LL, Braun TM, Zhang L, Cimprich B, Ronis DL, Mood DW. Assessing longitudinal quality of life in prostate cancer patients and their spouses: a multilevel modeling approach. Qual Life Res. 2011; 20:371-81. [PubMed: 20927648]

17. Ramsey SD, Zeliadt SB, Hall IJ, Ekwueme DU, Penson DF. On the importance of race, socioeconomic status and comorbidity when evaluating quality of life in men with prostate cancer. Journal of Urology. 2007; 177:1992-9. [PubMed: 17509278]

18. U.S. Census Bureau. Income, Poverty and Health Insurance in the United States: 2009. U.S. Department of Commerce; 2009.

19. Ware, JE.; Kosinski, M.; Keller, SD. SF-12®: how to score the SF-12® physical and mental health summary scales. Quality Metric Incorporated; Lincoln, RI: 1998.

20. Kroenke K, Spitzer RL, Williams JB. The Patient Health Questionnaire-2: validity of a two-item depression screener. Med Care. 2003; 41:1284-92. [PubMed: 14583691]

21. SAS Institute Inc. SAS 9.2 Cary. SAS Institute Inc; NC, USA: 2008.

22. Deimling GT, Sterns S, Bowman KF, Kahana B. The health of older-adult, long-term cancer survivors. Cancer Nursing. 2005; 28:415-424. [PubMed: 16330962]

23. Lev EL, Eller LS, Gejerman G, Lane P, Owen SV, White M, Nganga N. Quality of life of men treated with brachytherapies for prostate cancer. Health \& Quality of Life Outcomes. 2004; 2:28. [PubMed: 15198803]

24. Keating NL, Norredam M, Landrum MB, Huskamp HA, Meara E. Physical and Mental Health Status of Older Long-Term Cancer Survivors. Journal of the American Geriatrics Society. 2005; 53:2145-2152. [PubMed: 16398900]

25. Zebrack BJ, Yi J, Petersen L, Ganz PA. The impact of cancer and quality of life for long-term survivors. Psycho-Oncology. 2008; 17:891-900. [PubMed: 18050153]

26. Monahan PO, Champion V, Rawl S, Giesler RB, Given B, Given CW, Burns D, Bigatti S, Reuille $\mathrm{KM}$, Azzouz F. What contributes more strongly to predicting QOL during 1-year recovery from treatment for clinically localized prostate cancer: 4-weeks-post-treatment depressive symptoms or type of treatment? Qual Life Res. 2007; 16:399-411. [PubMed: 17091363]

27. Whyte EM, Rovner B. Depression in late-life: shifting the paradigm from treatment to prevention. Int J Geriatr Psychiatry. 2006; 21:746-51. [PubMed: 16858747]

28. Alibhai SM, Naglie G, Nam R, Trachtenberg J, Krahn MD. Do older men benefit from curative therapy of localized prostate cancer? J Clin Oncol. 2003; 21:3318-27. [PubMed: 12947068]

29. Baernholdt M, Hinton I, Yan G, Rose K, Mattos M. Factors associated with quality of life in older adults in the United States. Qual Life Res. 2012; 21:527-34. [PubMed: 21706127]

30. American Psychiatric Association. DSM; 2012. 
Table 1

Characteristics of Research Subjects, MEPS, 2009.

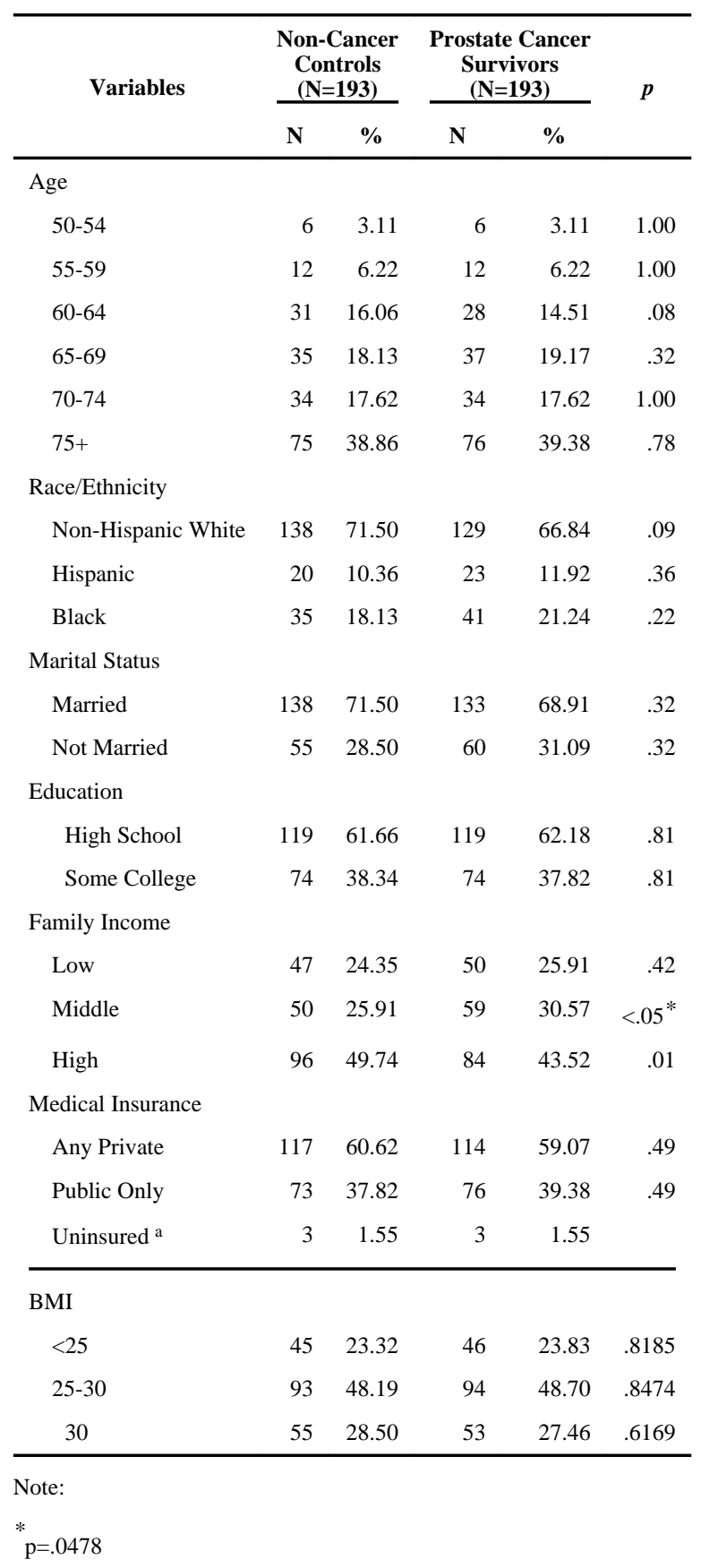


Table 2

Results of Descriptive Analysis

\begin{tabular}{|c|c|c|c|c|c|}
\hline \multirow[b]{2}{*}{ Variables } & \multicolumn{2}{|c|}{$\begin{array}{c}\text { Non-Cancer } \\
\text { Controls } \\
(\mathbf{N}=193) \\
\end{array}$} & \multicolumn{2}{|c|}{$\begin{array}{c}\text { Prostate Cancer } \\
\text { Survivors } \\
(\mathbf{N}=193) \\
\end{array}$} & \multirow[t]{2}{*}{$p$} \\
\hline & $\mathbf{N}$ & $\%$ & $\mathbf{N}$ & $\%$ & \\
\hline \multicolumn{6}{|l|}{ Health status } \\
\hline \multicolumn{6}{|l|}{ ADL } \\
\hline Need Help & 10 & 5.18 & 14 & 7.25 & .40 \\
\hline No & 183 & 94.82 & 179 & 92.75 & \\
\hline \multicolumn{6}{|l|}{$1 \mathrm{ADL}$} \\
\hline Need Help & 21 & 10.88 & 28 & 14.51 & .25 \\
\hline \multirow[t]{2}{*}{ No } & 172 & 89.12 & 165 & 85.49 & \\
\hline & Mean & SD & Mean & SD & $p>\mid \mathbf{z}$ \\
\hline Comorbidities & 2.78 & 2.06 & 3.25 & 2.05 & .02 \\
\hline Depressed Mood & 0.26 & 0.60 & 0.39 & 0.74 & .07 \\
\hline \multicolumn{6}{|l|}{ Quality of Life } \\
\hline Physical QOL & 45.45 & 11.42 & 42.72 & 11.33 & $<.01$ \\
\hline Mental QOL & 53.73 & 9.20 & 51.59 & 10.48 & .03 \\
\hline
\end{tabular}




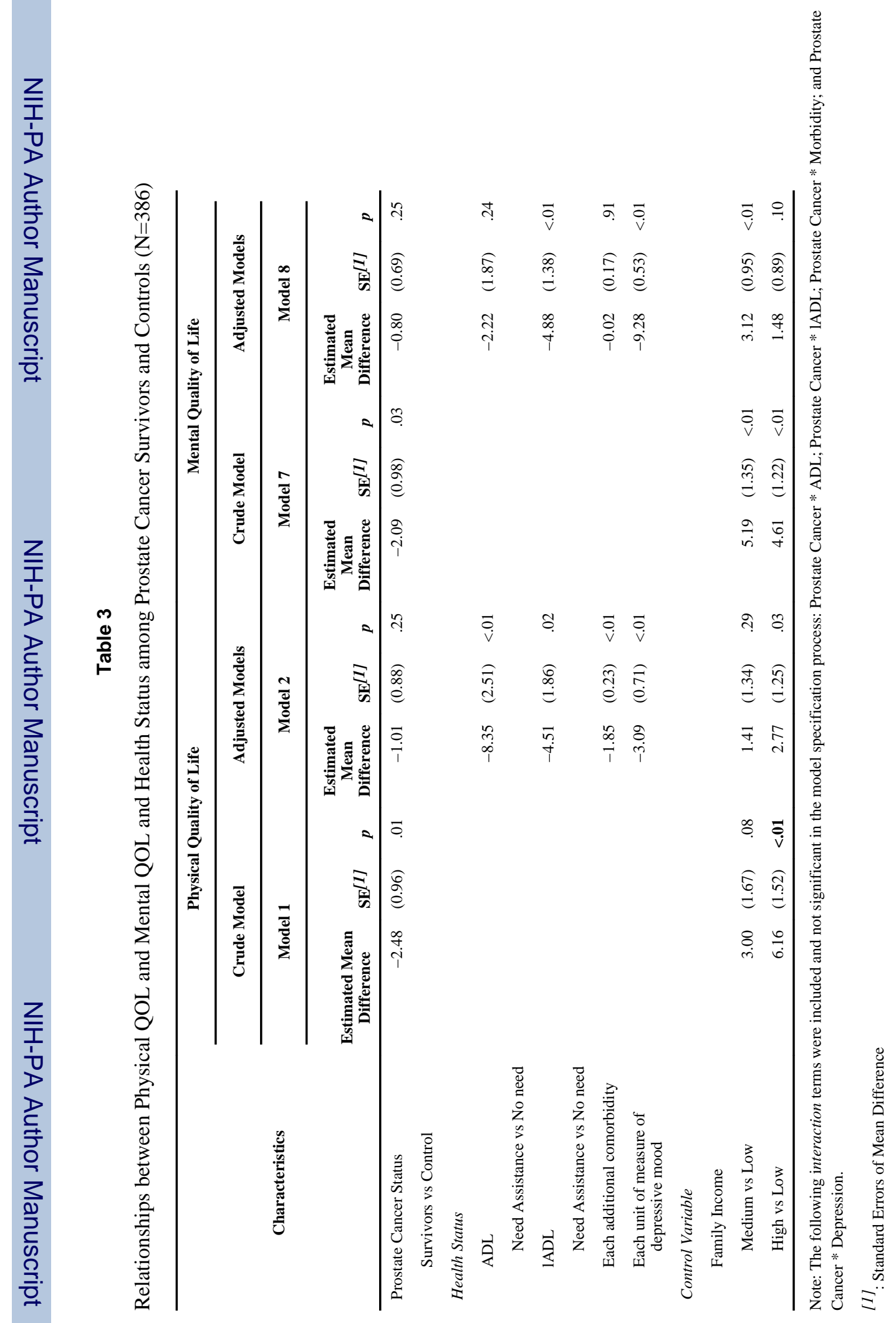

